\title{
A new twist in eutectic composition: deracemization of a racemic compound amino acid by Viedma ripening and temperature fluctuation.
}

\author{
Cristobal Viedma*[a], José Eugenio Ortiz ${ }^{[b]}$ \\ [a] Prof. C. Viedma Department of Crystallography and Mineralogy, University Complutense of \\ Madrid, E-mail: viedma@ucm.es \\ [b] Prof. J. E. Ortiz Universidad Politécnica de Madrid
}

\begin{abstract}
Viedma ripening and Temperature fluctuation are process based on solution phase racemization and dissolution-growth of racemic or scalemic conglomerates resulting in solid-phase deracemization. The dissolution-growth process is performed by abrasive grinding in the first case and by the temperature fluctuation in the second. But both methods have the intrinsic drawback of being only applicable to conglomerates, accounting for only $10 \%$ of all chiral molecules and are not suitable for the $90 \%$ of chiral compounds that crystallize as racemic compound. Herein we show that the enantiomeric excess in the eutectic point formed by a racemic compound and one of its enantiomers in suspension changes dramatically by continuous nucleation-growth-dissolution of the crystals, converting the racemic compound into the desire enantiomer. With this new finding the scope of Viedma ripening and temperature fluctuation could be significantly expanded and can shed new ideas about the origin of biological homochirality on earth.
\end{abstract}

Keywords: Amino acid, Chirality, Deracemization, Temperature fluctuation, Viedma ripening.

\section{Introduction}

The origin of biological homochirality, exclusively left-handed amino acids and right-handed sugars, remains as one of the most fundamental question and a fascinating aspect of prebiotic chemistry in the origin of life. ${ }^{[1]}$

Processes that can produce states of broken chiral symmetry are of particular interest to chemistry, physics, and biology. Several mechanisms that lead to an imbalance between enantiomers have been proposed together with an amplification mechanism as an explanation for the origin of biomolecular single handedness. ${ }^{[2-4]}$

On the molecular level, chirality has a profound impact on recognition and interaction events and is thus important to biochemistry and pharmacology. ${ }^{[5]}$

As a result of regulatory aspects, more and more efforts are put into bringing enantiopure medicines to the market. In many cases this can be achieved by an 
enantioselective synthesis. Nevertheless, in an increasing number of cases, this would involve too many synthetic steps for the process to be economically viable.

Chiral separation by crystallization can be an alternative. In addition, these protocols simplify enormously the production of enantiomerically pure substances and can be adapted to the pharmaceutical industry to get chiral drugs.

In 2005, Viedma demonstrated that continuous abrasive grinding of a racemic suspension of $d$ - and I-NaCLO3 conglomerate crystals under a near-equilibrium condition, transform the crystals in the suspension into an enantiopure state. ${ }^{[3]}$

Three years after, Noorduin et al. were able to extend this process to intrinsically chiral organic compounds crystallizing as conglomerates and undergoing fast racemization of the pair of chiral molecules within the solution phase of the suspension. They showed that the process can be applied to amino acid derivatives, metal-organic complexes and isoindolinones. ${ }^{[6]}$

Currently, grinding-induced deracemization is being explored as a practical route to produce enantiomerically pure compounds that can be used in the manufacture of pharmaceuticals. It also provides a scenario for the evolution of single chirality as is found in nature.

In 2007, we suggested [7] that "a growth-dissolution process enhanced by temperature fluctuation (or dry-wet cycles) in a system with a racemic suspension in solution of amino acids conglomerate crystals, or polymers, with racemization of molecules in solution could give complete chiral purity and may have played a key role in the origin of biological homochirality on Earth."

One year after, 2008, we reported the first example of the evolution of total enantio-enrichment of the solid phase for the conglomerate of an essential amino acid mediated by solution-phase racemization in the absence of the attrition enhancement by a strong temperature gradient. ${ }^{[8]}$

In 2011, total deracemization by temperature fluctuation or temperature cycling was demonstrated by some of us in a suspension of NaCLO3. ${ }^{99}$ For this, a difference of $14^{\circ} \mathrm{C}$ was established between the lower part and the upper part of a flask, initiating a dissolution-growth process of the crystals that finally generated a homochiral system (figure 1) It was shown that the homogenization of the temperature in the flask by strong agitation produced the disappearance of the deracemization phenomenon, demonstrating, unequivocally, that the driving force of the deracemization process was the fluctuation or cycles of temperature. Therefore, temperature fluctuation was shown to be a viable and efficient alternative to traditional attrition deracemization. 


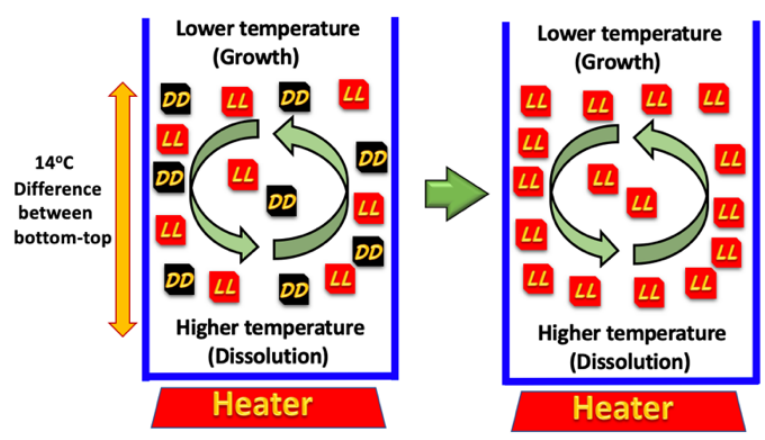

\section{Figure 1}

Deracemization by temperature fluctuation $\left(14^{\circ} \mathrm{C}\right)$ of a racemic suspension of $\mathrm{NaCLO} 3$ crystals.

In 2013 Suwannsang et al. [10] reported other example on the effect of temperature fluctuations with racemization in solution, using programmed heating-cooling cycles, under near-ambient conditions, on the deracemization of a model compound, 1- (4-chlorophenyl) -4,4-dimethyl-2- (1H-1,2,4-triazol-1-yl) pentan-3- 1.

A technical modification was introduced in this work and the entire system experienced intermittent temperature rises and falls, meaning that the temperature fluctuation occurred in time and not in space.

More recently, another modification returned to the fluctuation of temperature in space, although not within the same system but in two different intercommunicating systems with different temperatures. ${ }^{[11]}$

Obviously the deracemization through these two technical modifications take advantage of the same experimentally established principle, ${ }^{[8-9]}$ i.e. homochirality can ultimately be achieved through a dissolution-growth process maintained by fluctuations in temperature or thermal cycles.

In some way, this new method could be considered as a derivative of the classic Viedma deracemization in which the dissolution-growth generated by grinding now occurs through temperature fluctuation.

Although being promising methods for deracemization, Viedma Ripening and the temperature cycling method have the intrinsic drawback of being only applicable to conglomerates. A rough estimate tells us that less than $10 \%$ of chiral compounds crystallize as conglomerates (the two enantiomers crystallize into separate crystals). ${ }^{[12]}$ This restriction excludes the utilization of these deracemization methods for roughly $90 \%$ of the known chiral molecules since these compounds crystallize as racemic compounds, (both enantiomers crystallize in the same crystal). ${ }^{[12]}$

The deracemization of racemic compounds by grinding or temperature fluctuation, therefore, constitutes a great challenge and above all, considering prebiotic chemistry, the deracemization of the main chiral biological molecules 
such as amino acids. Only 2 of the 20 proteinogenic amino acids are reported to form a racemic conglomerate (asparagine and threonine).

Here we report the deracemization in water of the proteinogenic amino acid aspartic acid, a racemic compound, using the combination of concepts behind Viedma ripening and temperature fluctuation. We show herein that the continuous nucleation-growth-dissolution of the crystals through grinding, temperature fluctuation and racemization in solution generates a drastic change in the enantiomeric excess (ee) at the eutectic point of a crystalline suspension in which the racemic compound and one of its enantiomers are present in the solid phase. This alteration of the ee at the eutectic is the driving force behind the deracemization process and broadens the possibility of deracemization of racemic compounds by combining these two techniques.

\section{Experimental}

For the deracemization of aspartic acid a suspension of LD aspartic acid crystals $(1600 \mathrm{mg})$ and $\mathrm{L}$ or $\mathrm{D}$ aspartic acid crystals $(1600 \mathrm{mg})$ in a flask of $25 \mathrm{~mL}$ with water $(22 \mathrm{~mL})$ in the presence of $3 \mathrm{~mm}$ glass beads $(8 \mathrm{~g}$.). The suspension was strongly stirred (800 rpm) in order to homogenize the size of the crystals during 1-2 hours. After homogenization the solution phase racemization was initiated by adding sodium hydroxide $(20 \mathrm{mg})$ and then was gently stirred (120 rpm). The flask was placed on a heater generating a temperature difference between the bottom of the flask (in contact with the heating plate) and the top of the flask (in contact with the atmosphere at room temperature) of approximately $14-18{ }^{\circ} \mathrm{C}$. We have used a fan to aerate the top of the flask and help establish a proper temperature fluctuation.

The temperature range of the experiments is of great importance in the deracemization time. We have chosen temperatures that allow deracemization in a few hours $\left(78^{\circ} \mathrm{C}-60^{\circ} \mathrm{C}, 24\right.$ hours, $68^{\circ} \mathrm{C}-50^{\circ} \mathrm{C}, 48$ hours). By increasing the racemization agent, the deracemization time could decrease significantly, but the yield is lower because more product remains in solution.

Samples of the solid phase were collected over time and enantiomeric purity was measured using chiral HPLC methods. The final product was verified by X-ray powder diffraction (XRPD) (see Supporting Information).

\section{Results and Discussion}

We observed in all the systems that the continuous nucleation-growth-dissolution of the crystals through temperature fluctuation and racemization in solution leads to a rise in ee of solid phase in time. (Figure 2) 


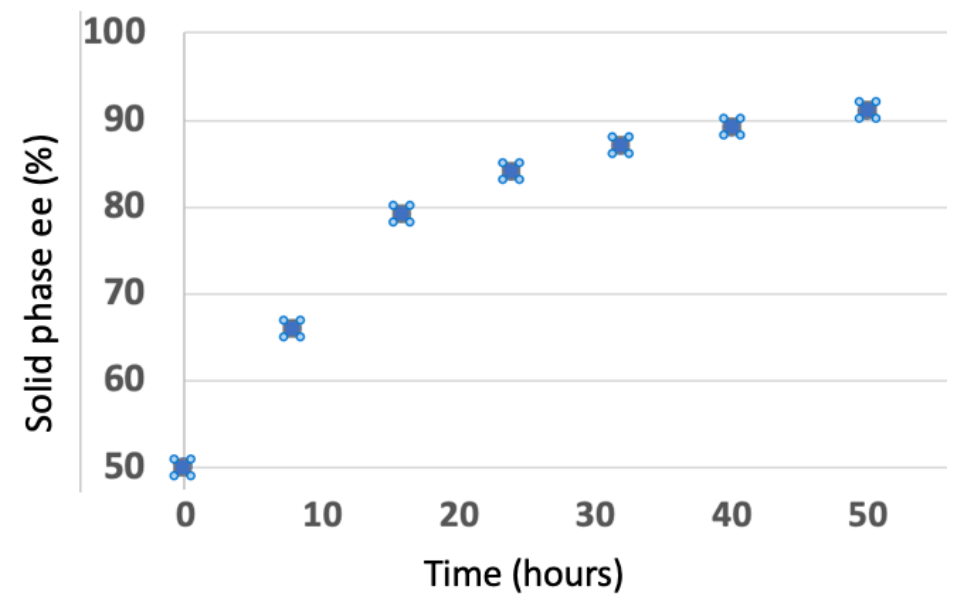

Figure 2

Evolution of solid-phase ee for racemic compound aspartic acid and one enantiomer with solution-phase racemization.

Furthermore, under the same conditions, experiments were carried out, in which suspensions of equal amounts of racemic compound and now with both enantiomers present in the system were stirred for two days. The final solid phase was characterized by X-ray powder diffraction (XRPD), corresponding to the racemic compound. The incorporation of the two enantiomers in the racemic phase shows that the aspartic acid, racemic compound, is in fact the most stable phase at these temperatures and conditions, which contrasts with the result obtained when only one enantiomer is present (Figure 3).

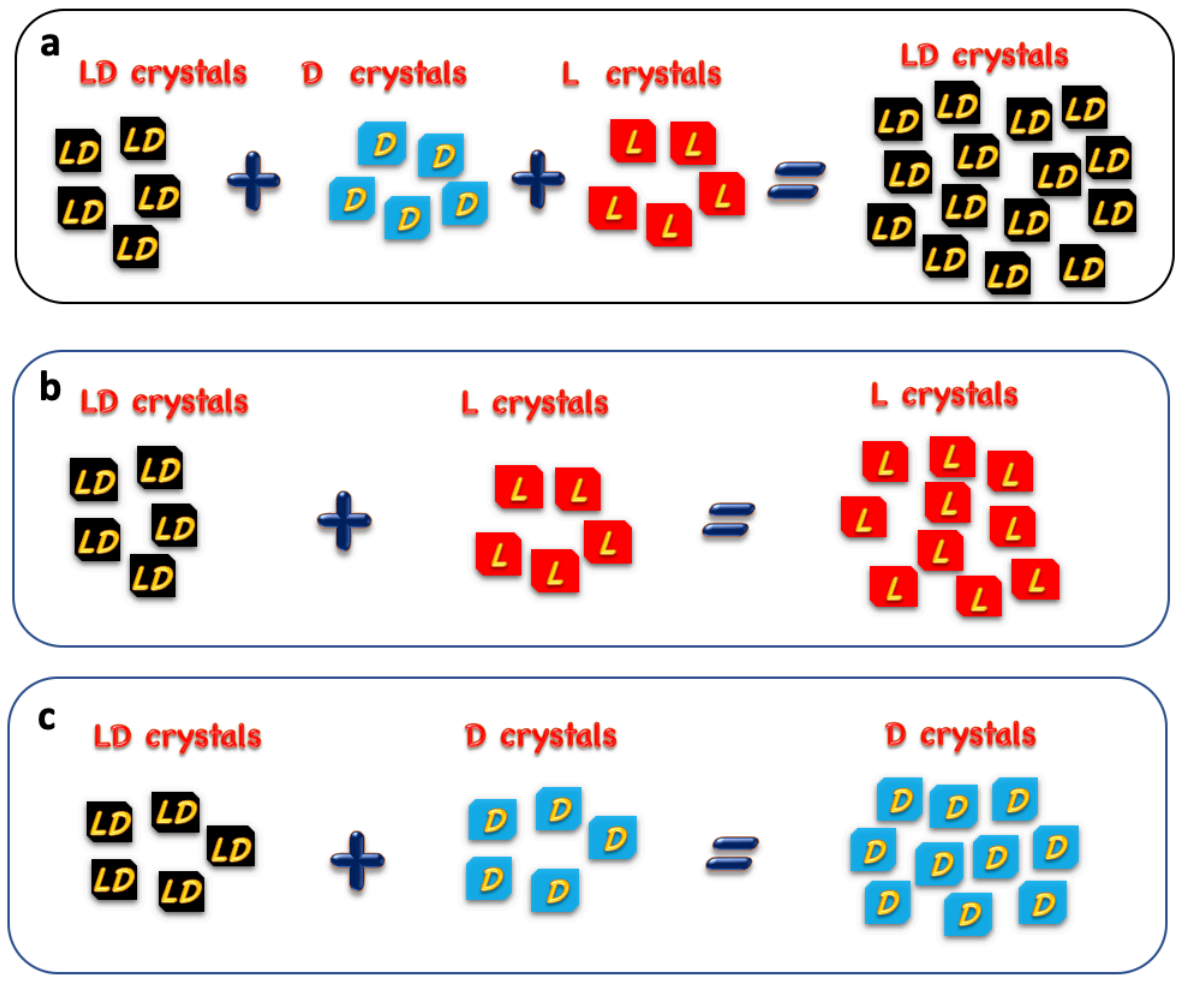

Figure 3

Evolution of three experiments following the protocol established in the text. 
(a) the two enantiomers are present in the system with the racemic compound. Finally, the entire product is a racemic compound, that is, the stable phase is the racemic compound

(b) Only the $L$ enantiomer is present in the system with the racemic compound. Finally, all the product belongs to the enantiomer $L$.

(c) Only the $D$ enantiomer is present in the system with the racemic compound. Finally, the entire product belongs to the enantiomer D.

At the eutectic point, where the saturated solution and the two enantiomeric crystals coexist in equilibrium, the composition of the solution of the chiral substance is fixed at a given temperature ${ }^{[12]}$. That is, initially even if the $D$ and $L$ chiral crystals differ in amount, the solution phase will contain identical concentrations of each chiral molecule. For a racemic conglomerate, thermodynamics predicts that the supernatant solution phase is racemic for any solid composition Therefore, at equilibrium a conglomerate always exhibits a solution ee of $0 \%$ (Figure 4 ).

During the process of Viedma ripening a slurry of the conglomerate crystals in a saturated solution is intensively ground to promote breakage and dissolution as well as growth of the crystals. The grinding increases the number of small clusters, and enhances the cluster incorporation to crystals of the same phase.

Clusters or subcritical crystals of the major enantiomer in the solution are less likely to dissolve than those of the minor enantiomer, because they are more likely to be rescued from dissolution by fusing with the more prevalent and abundant crystals of the same hand while the clusters of the minority solid phase have a lower probability of encountering a crystal of the same handedness ${ }^{[13]}$

In this way the clusters of the minor enantiomer are more abundant in solution and therefore dissolves more quickly, then the solution ee is found to be enriched in the enantiomer that forms the minor population in the solid phase.

This was demonstrated experimentally, and without racemization during the grinding of a slurry, the solution contains an enantiomeric excess that is the inverse of that in the solid phase. ${ }^{[13]}$

In the presence of a racemization catalyst, this inverted enantiomeric excess in the solution phase erodes, becoming the driving force for the deracemization process by means of a net flux of molecules from crystals of the minor handedness to crystals of the handedness that forms the major population in the solid phase. In this way, an enantiopure crystalline final state can be achieved, in up to $100 \%$ yield. ${ }^{[13]}$

But the experiments in this work are different, they start from systems in which the racemic compound and one of its enantiomers are present in the solid phase, coexisting in equilibrium with the saturated solution from the beginning, this mixture have received little attention until Blackmon's seminal work ${ }^{[14]}$. The minor enantiomer can be present in solution only via dissolution from the solid racemic compound. 
Therefore, the ee in the solution at equilibrium is controlled by the relative solubility of the racemic compound and the enantiopure crystals and this ee can fall between 0 and $100 \%$ ee. ${ }^{[15]}$ In the case of aspartic acid, the eutectic ee has a value of approximately $55 \%$, ee of the common chiral molecule in the two phases, the L-molecule in this example. (Figure 4)

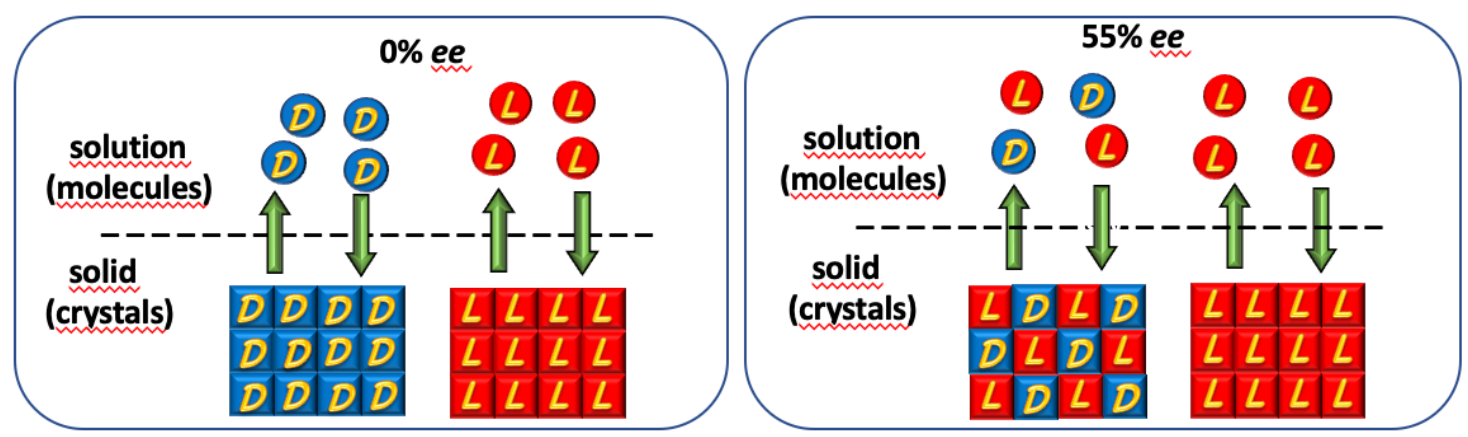

\section{Figure 4}

(a) For a racemic conglomerate the supernatant solution phase is racemic $(0 \%$ ee at the eutectic). (b) For a racemic compound coexisting in equilibrium with one of its enantiomers, the ee in solution is controlled by the relative solubility of the racemic compound and the enantiopure crystals ( $55 \%$ for aspartic acid)

It is evident that, in the present case, the eutectic composition formed for the enantiomer and the racemic compound is always thermodynamically unfavorable for the deracemization of the racemic compound in a racemizing medium. In stagnant conditions there would be a permanent flow of molecules $L$ towards $D$ (entropy) and therefore the enantiomer crystals would disappear feeding the racemic compound.

But, generating a strong nucleation-growth-dissolution process through grinding and temperature fluctuation, deracemization could still be achieved using the kinetic advantage provided by the enantiomeric excess of the eutectic that kinetically favors the growth of the enantiomer crystals and the appearance of clusters of the molecule in excess, as a consequence of the fact that minority molecules in solution, belonging only to the racemic compound, are less likely to form clusters or find the appropriate structural position in the crystals already present.

To test this, additional experiments were performed under the same conditions but without racemization. Samples of the solution were taken two hours after the start of the experiments to measure the possible evolution of the ee. We found that the liquid phase surprisingly approaches $0 \%$ ee when at the beginning of the experiment, in the equilibrium of both solid phases, the enantiomeric excess of the eutectic was $55 \%$ (Figure 5). This shows that the rapid formation and growth of crystals depletes the initially largest population of molecules in the solution. Both populations of chiral molecules tend to equalize. Furthermore, the kinetic advantage of an easier incorporation of the common molecules to the enantiomer and the racemic compound is maintained due to the greater number of equivalent positions on the surface of the crystals ${ }^{[16]}$ 


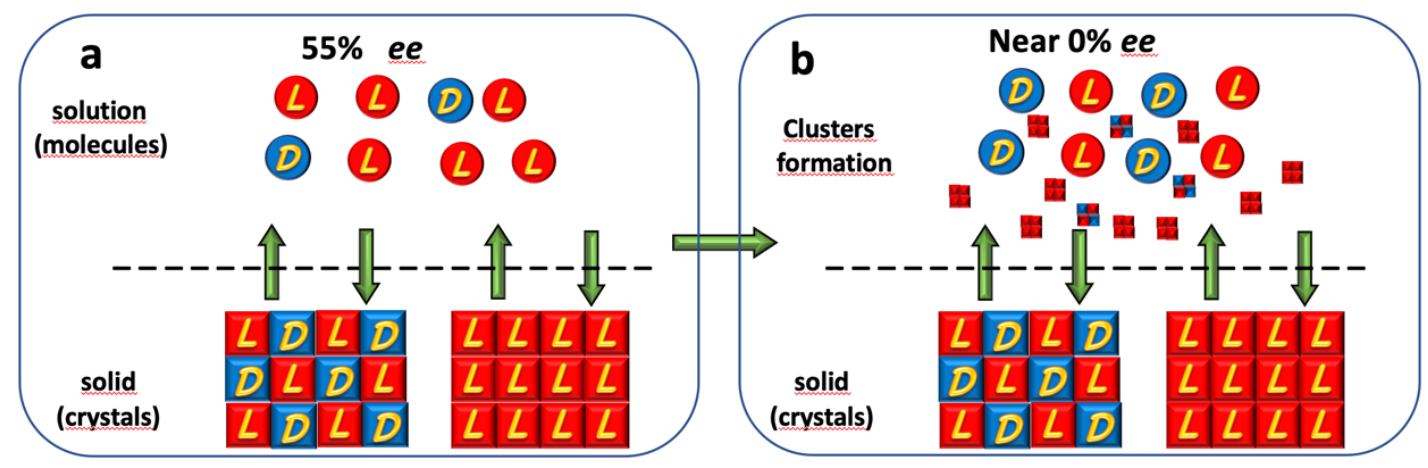

\section{Figure 5}

(a) Racemic crystals of aspartic acid coexisting in equilibrium with one of its enantiomers have an ee in solution of $55 \%$.

(b) Racemic crystals of aspartic acid coexisting in equilibrium with one of its enantiomers under nucleation conditions show an ee close to cero.

The solid phase replenishes the solution phase constantly. Under racemizing conditions, this permanent kinetic disequilibrium, with a more rapid incorporation of the enantiomer molecules, allows a net flux of molecules from the racemic compound to the enantiomer by an adequate balance between growth rate and the racemization in solution.

Thus, the final result is a conversion of the crystals of the racemic compound, which serves as a gradual feeding, into crystals of the enantiomer present in the system in contrast to Viedma ripening in which only the opposite enantiomer serves as food. This is so, not only when the quantity of the enantiomer-racemic compound, in solid state, is the same (1: 1), but in a higher proportion for the racemic compound (1:1.5) the system advances towards homochirality, although much slower (Figure 6).

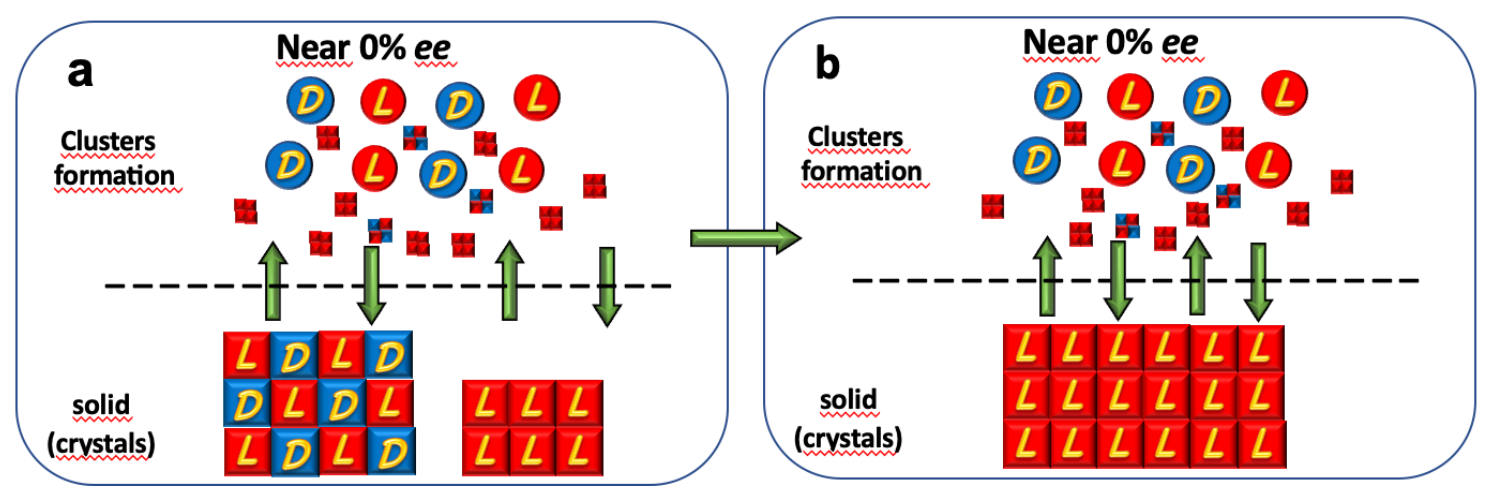

\section{Figure 6}

(a) Racemic aspartic acid crystals that coexist in equilibrium with crystals of one of its enantiomers under nucleation conditions present an ee close to zero, not being necessary an equality in the quantity of both solid phases.

(b) The crystals of the racemic compound serve as a gradual feed for crystals of the enantiomer present in the solution. 
A big difference between the standard Viedma ripening and these experiments is that in the first case, clusters are produced from the solid phase, whereas now clusters arise primarily from the core of the solution, so grinding may not be absolutely essential in some cases, but it profoundly helps growth-dissolution kinetics, and kinetics is the original source of the driving force for this deracemization process

With this new finding, deracemization of a solid phase might not be restricted to $10 \%$ of chiral molecules that crystallize as a conglomerate since this method could be extended to some other racemic compounds. For this, it would be necessary to choose the appropriate solvent, racemization agent, temperature fluctuation, cooling rate and hope that both phases are not separated by too large an energy gap.

When a compound is stable as racemic compound, deracemization could still be achieved, at least in some cases, using the perpetual struggle between thermodynamics and kinetics.

\section{References}

[1] W. A. Bonner, Origins Life Evol. Biospheres 1991, 21, 59-111.

[2] F. C. Frank, M. Biochimica et Biophysica Acta 1969, 11, 459-463

[3] C. Viedma, Physical Review Letters 2005, 94, 065504

[4] K. Soai, T. Shibata, H. Morioka, K. Choji, Nature, 1995, 374, 767-768

[5] I. Agranat, H. Caner, Drug Discov. Today 1999, 4, 313

[6] W. Noorduin, T. Izumi, A. Millemaggi, M. Leeman, H. Meekes, W. Van Enckevort, R. Kellogg, B. Kaptein, E. Vlieg, D. Blackmond, J. Am. Chem. Soc. 2008, 130 (4), 1158-1159

[7] C. Viedma, Astrobiology 2007, 7 (2), 312-319

[8] C. Viedma, J. Ortiz, T. Torres, T. Izumi, D. Blacmond, J. Am. Chem. Soc. 2008, 130, (46), 15274-15275

[9] C. Viedma, P. Cintas, Chemical Communications 2011, 47, 12786-12788 [10] K. Suwanna, A. E. Flood, C. Rougeot, G. Coquerel, Cryst. Growth Des. 2013, 13, 3498-3504;

[11] K. Suwannasang, A. Flood, G. Coquerel, Cryst. Growth Des. 2016, 16, 11 , 6461-6467

[12] J. Jacques, A. Collet, S. Wilen, 1991, Enantiomers, Racemates and Resolutions, Krieger Publishing Company, Florida, 447.7

[13] W. Noorduin, W. Enckevort, H. Meekes, B. Kaptein, R. Kellogg, J. Tully, M. McBride, E. Vlieg, Angewandte 2010, 49, (45), 8435-8438

[14] M. Klussmann, H. Iwamura, S. Mathew, D.Well, U. Pandya, A. Armstrong,

D. Blackmond, Nature 2006, 441, 7093, 621-623

[15] M. Klussmann, A. J. R. White, A. Armstrong, D. G. Blackmond, Angew. Chem. Int. Ed. 2006, 45, 7985-7989

[16] J.M. Garcia-Ruiz, J.L. Amorós, Estudios Geológicos 1980, 36, 193-200 
Competing interest statement: Complutense University of Madrid has applied for a patent (P202130729) on the use of this deracemization method with C.V.as inventor 